\title{
pra gMATIZES
}

Ano VII $n^{\circ} 12$ - out/2016 a mar/2017 www.pragmatizes.uff.br

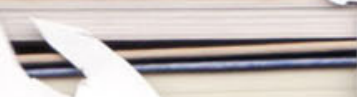

DOSSIÊ "LEITURAS DO MUNDO: FORMAS DE

Carnet de routes: following Blaise Cendrars in Brazil EXPRESSÃO CRIATIVAS E COMUNICATIVAS" - $=$ Diário de bordo: seguindo Blaise Cendrars no Brasil DOSSIER “WORLD'S LECTURES: FORMS OF

LUCA FORCUCCI EXPRESSION CREATIVITY AND COMMUNICATION"

\section{Apresentação do Dossiê}

Dossier's presentation

MARISA MELLO e RÔSSI GONÇALVES, editoras

Rodas culturais, UPP, funk e milícias:

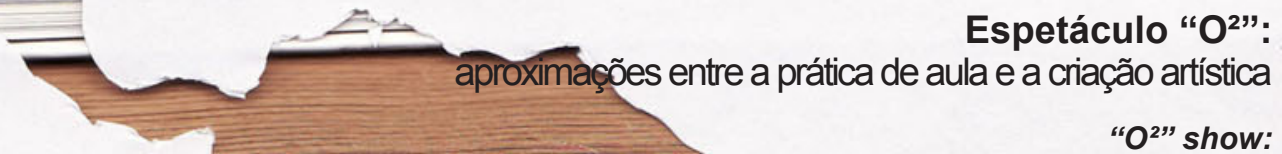
uma análise da cultura urbana carioca frente às políticas de segurança e às organizações criminosas

Cultural Circles, UPP, Funk and Militia: an analysis of Rio de Janeiro's urban culture security policies and criminal organizations

Cultura: a dádiva da sociedade ROSSI GONÇALVES e GUILHERME SANTOS

Culture: the social's gift

approximation between class practice and artistic creation LARA SEIDLER DE OLIVEIRA

IO AURELIO VIANNA LOPES

O sarau como estratégia de resistência poética reflexão sobre novos territórios culturais

Soiree as poetic resistance strategy and reflection on cultural new territories

IDEMBURGO FRAZÃO

Torcedores organizados: enigma como contrapeso ao fantasma da razão esclarecida

Football supporters groups:

enigma as counterweight to the ghost of the enlightened reason GUSTAVO COELHO

Cidade Maravilhosa:

O Rio de Janeiro representado pelas letras

Wonderful City: Rio de Janeiro represented by the letters PRISCILLA OLIVEIRA XAVIER

Práticas de leitura na contemporaneidade: experiências em bibliotecas na cidade do Rio de Janeiro

Reading practices in contemporaneity:

\section{$\epsilon$} O jornalista e o assessor de imprensa no cinema noir através do filme A Embriaguez do Sucesso

The Journalist and the PR professional in Film Noir in the movie Sweet Smell of Success the movie Sweet Smell of Success ALEX SAIMPAIO PIRES e MICHELE NEGRINI Seguindo a constituição da Joalheria contemporânea

Following the constitution of the Contemporary Jewelery ANA NEUZA BOTELHO VIDELA libraries' experiences in the city of Rio de Janeiro

MARISA S. MELLO

ENSAIO ESSAY

Mudar de corpo em ode aos invisíveis

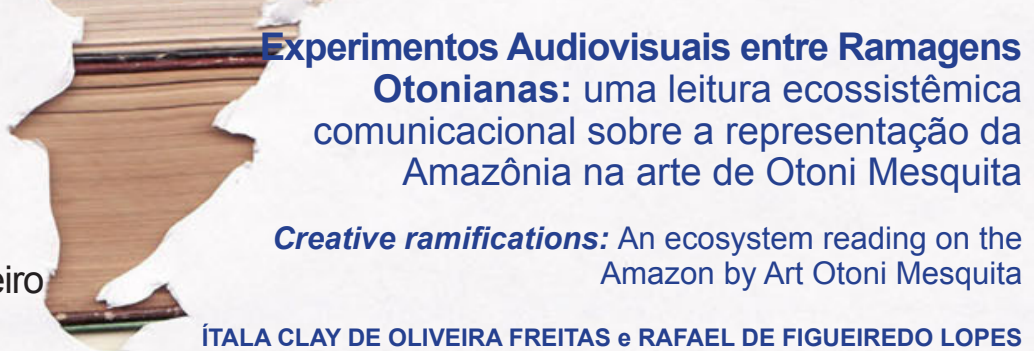
ÍTALA CLAY DE OLIVEIRA FREITAS e RAFAEL DE FIGUEIREDO LOPES

(a)

\section{Changing body in ode to the invisible ones}




\section{PragMATIZES \\ Revista Latino Americana de Estudos em Cultura}

\section{Ano VII no 12 - out/2016 a mar/2017}

EDITORES

1. Flávia Lages, Universidade Federal Fluminense, Instituto de Arte e Comunicação Social, Departamento de Arte, Curso de Produção Cultural, Brasil 2. Luiz Augusto Rodrigues, Universidade Federal Fluminense, Instituto de Arte Comunicação Social, Departamento de Arte, Curso de Produção Cultural, Brasil 3. Ana Enne, Universidade Federal Fluminense, Instituto de Arte e Comunicação Social, Departamento de Estudos de Mídia, Brasil

\section{CONSELHO EDITORIAL}

1. Adriana Facina, Universidade Federal do Rio de Janeiro, Museu Nacional, Brasil 2. Christina Vital, Universidade Federal Fluminense, Departamento de Sociologia, Brasil 3. Danielle Brasiliense, Universidade Federal Fluminense, Departamento de Comunicação, Brasil

4. João Domingues, Universidade Federal Fluminense, Instituto de Arte e Comunicação Social, Departamento de Arte, Curso de Produção Cultural, Brasil 5. José Maurício Saldanha Alvarez, Universidade Federal Fluminense,

Departamento de Estudos de Mídia, Brasil

6. Leandro Riodades, Universidade Federal Fluminense, Departamento de Artes e Estudos Culturais, Brasil

7. Leonardo Guelman, Universidade Federal Fluminense, Departamento de Arte, Brasil 8. Lívia de Tommasi, Universidade Federal Fluminense, Departamento de Sociologia, Brasil

9. Lygia Segala, Universidade Federal Fluminense, Departamento de

Fundamentos Pedagógicos, Brasil

10. Marildo Nercolini, Universidade Federal Fluminense, Departamento de 10. Marildo Nercolini, Unives de Mídia, Brasil

Estudos de Mídia, Brasil
11. Paulo Carrano, Universidade Federal Fluminense, Departamento Sociedade, 11. Paulo Carrano, Universidade Fed
Educação e Conhecimento, Brasil

Educação e Conhecimento, Brasil
12. Rossi Alves, Universidade Federal Fluminense, Departamento de Artes e Estudos Culturais, Brasil

13. Wallace de Deus Barbosa, Universidade Federal Fluminense, Departamento de Arte, Brasil

\section{COMITÊ EDITORIAL}

1. Adair Rocha, Universidade do Estado do Rio de Janeiro e Pontifícia Universidade Católica do Rio de Janeiro, Departamento de Comunicação Social, Brasil

2. Alberto Fesser, Socio Director de La Fabrica em Ingenieria Cultural / Director de La Fundación Contemporánea, Espanha

3. Alessandra Meleiro, Universidade Federal de São Carlos, Brasil

4. Alexandre Barbalho, Universidade Estadual do Ceará e Universidade Federal

do Ceará, PPG Cultura e Sociedade, Brasil

do Ceará, PPG Cultura e Sociedade, Brasil

5. Allan Rocha de Souza, Universidade Federal Rural do Rio de Janeiro,
UFRJ/PPG em Políticas Públicas, Estratégias e Desenvolvimento, Brasil

UFRJ/PPG em Políticas Públicas, Estratégias e Desenvolvimento, Brasil
6. Angel Mestres Vila, Universitat de Barcelona, Master en Gestión Cultural /

Director geral de Transit projectes, Espanha

7. Antônio Albino Canela Rubin, Universidade Federal da Bahia, Instituto de

Humanidades, Artes e Ciências / Pesquisador do CNPq, Brasil

8. Carlos Henrique Marcondes, Universidade Federal Fluminense, Departamento

de Ciência da Informação, Brasil

9. Cristina Amélia Pereira de Carvalho, Universidade Federal do Rio Grande do

Sul, Departamento de Administração / Pesquisadora do CNPq, Brasil

10. Daniel Mato, Universidade Nacional Tres de Febrero, Instituto

Interdisciplinario de Estudios Avanzados/CONICET: Consejo Nacional de Investigaciones Científicas y Técnicas, Argentina

11. Eduardo Paiva, Universidade Estadual de Campinas, Departamento de Multimeios, Mídia e Comunicação, Brasil

Multimeios, Mídia e Comunicação, Brasil

12. Edwin Juno-Delgado, Université de Bourgogne / ESC
Paris, Faculdad Gestión, Derecho y Finanzas , França

Paris, Faculdad Gestión, Derecho y Finanzas, França
13. Fernando Arias, Observatorio de Industrias Creativas de la Ciudad de

13. Fernando Arias, Obser
Buenos Aires, Argentina

14. Gizlene Neder, Universidade Federal Fluminense, PPG em História, Brasil

15. Guilherme Werlang, Universidade Federal Fluminense, Departamento de Arte, Brasil

16. Guillermo Mastrini, Universidad Nacional de Quilmes, Maestría en Industrias

Culturales, Argentina

17. Hugo Achugar, Universidad de la Republica, Uruguai

18. Isabel Babo - Universidade Lusófona do Porto, Portugal

19. Jaime Ruiz-Gutierrez, Universidad de los Andes, Colombia

20. Jeferson Francisco Selbach, Universidade Federal do Pampa, curso de

Produção e Política Cultural, Brasil
21. José Luis Mariscal Orozco, Universidad de Guadalajara, Instituto de Gestion del conocimiento y del aprendizaje en ambientes virtuales, México

22. José Márcio Barros, Pontifícia Universidade Católica de Minas Gerais, PPG em Comunicação, Brasil

23. Julio Seoane Pinilla, Universidad de Alcalá, Master Estudios Culturales, Espanha 24. Lia Calabre, Fundação Casa de Rui Barbosa, Brasil

25. Lilian Fessler Vaz, Universidade Federal do Rio de Janeiro, PPG em Urbanismo, Brasil

26. Lívia Reis, Universidade Federal Fluminense, Instituto de Letras, Brasil 27. Luiz Guilherme Vergara, Universidade Federal Fluminense, Departamento 27. Luiz Guilhen

28. Manoel Marcondes Machado Neto, Universidade do Estado do Rio de 28. Manoel Marcondes Machado Neto, Universidade do Estad
Janeiro, Departamento de Ciências Administrativas, Brasil

Janeiro, Departamento de Ciências Administrativas, Brasil 29. Márcia Ferran, Universidade Federal Fluminense, Departamento de Artes e Estudos Culturais, Brasil

30. Maria Adelaida Jaramillo Gonzalez, Universidad de Antioquia, Colômbia

31. Maria Manoel Baptista, Universidade de Aveiro, Departamento de Línguas e Culturas, Portugal

32. Marialva Barbosa, Universidade Federal do Rio de Janeiro, Escola de Comunicação / Pesquisadora do CNPq, Brasil

33. Marta Elena Bravo, Universidad Nacional de Colombia - sede Medellín, Profesora jubilada y honoraria da Faculdad de Ciencias Humanas y Económicas, Colombia 34. Martín A. Becerra, Universidad Nacional de Quilmes / CONICET: Consejo Nacional de Investigaciones Científicas y Técnicas, Argentina

35. Mónica Bernabé, Universidad Nacional de Rosario, Maestria en Estudios Culturales, Argentina

36. Muniz Sodré, Universidade Federal do Rio de Janeiro, Escola de 36. Muniz Sodré, Universidade Federal do Rio

Comunicação / Pesquisador do CNPq, Brasil
37. Orlando Alves dos Santos Jr., Universidade Federal do Rio de Janeiro,

37. Orlando Alves dos Santos Jr., Universidade Federal do Rio
Instituto de Pesquisa e Planejamento Urbano e Regional, Brasil

38. Patricio Rivas, Escola de Gobierno de la Universidad de Chile, Chile 39. Paulo Miguez, Universidade Federal da Bahia, Instituto de Humanidades, Artes e Ciências, Brasil

40. Ricardo Gomes Lima, Universidade Estadual do Rio de Janeiro,

Departamento de Artes e Cultura Popular, Brasil

41. Stefano Cristante, Università del Salento, Professore associato in Sociologia dei processi culturali, Italia

42. Teresa Muñoz Gutiérrez, Universidad de La Habana, Profesora Titular del Departamento de Sociologia, Cuba

43. Tunico Amâncio, Universidade Federal Fluminense, Departamento de Cinema, Brasil 44. Valmor Rhoden, Universidade Federal do Pampa, curso de Relações Públicas [com ênfase em Produção Cultural], Brasil Públicas [com ênfase em Produção Cultural], Brasil
45. Victor Miguel Vich Flórez, Pontifícia Universidad Católica del Perú, Maestría de Estudios Culturales, Peru

46. Zandra Pedraza Gomez, Universidad de Los Andes / Maestria em Estudios Culturales, Colômbia

\section{EDITORES ASSOCIADOS JUNIOR:}

1. Bárbara Duarte, doutoranda em Sociologia, Universidade Federal da Paraíba 2. Deborah Rebello Lima, mestranda em História, Política e Bens Culturais pelo CPDOC, Fundação Getúlio Vargas / pesquisadora pela Fundação Casa de Rui Barbosa 3. Gabriel Cid, doutorando em Sociologia pelo Instituto de Estudos Sociais e Políticos, Universidade do Estado do Rio de Janeiro

4. Leandro de Paula Santos, doutorando em Comunicação pela ECO, Universidade Federal do Rio de Janeiro

5. Marine Lila Corde, doutoranda em Antropologia Social pelo Museu Nacional, 5. Marine Lila Corde, doutoranda em Antro

6 . Sávio Tadeu Guimarães, doutorando em Planejamento Urbano e Regional 6. Sávio Tadeu Guimarães, doutorando em Planejam

pelo IPPUR, Universidade Federal do Rio de Janeiro lica do Rio de Janeiro / professora de Direito Ambiental (PUC-Rio)

\section{CRIADOR DA MARCA}

Laert Andrade

DIAGRAMAÇÃO:

Ubirajara Leal
REALIZAÇÃO:

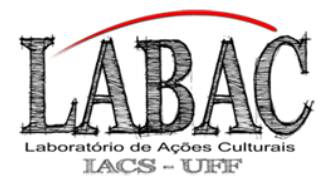

PPuI

APOIO:

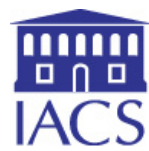

PARCEIROS:
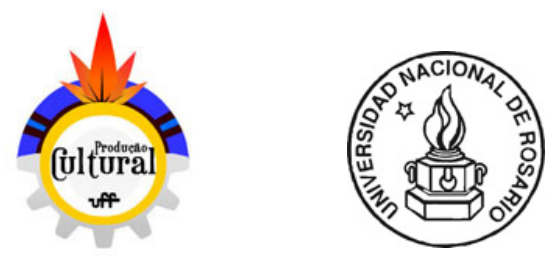

Maestría en Estudios Culturales 
PragMATIZES - Revista Latino Americana de Estudos em Cultura.

Ano VII no 12, (OUT/2016 a MAR/2017). - Niterói, RJ: [s. N.], 2016.

(Universidade Federal Fluminense / Laboratório de Ações Culturais LABAC)

Semestral

ISSN 2237-1508 (versão on line)

1. Estudos culturais. 2. Planejamento e gestão cultural.

3. Teorias da Arte e da Cultura. 4. Linguagens e expressões artísticas. I. Título. 


\section{Sumário / Summary}

\section{APRESENTAÇÃO DO DOSSIÊ / DOSSIER'S PRESENTATION}

DOSSIÊ: "Leituras do mundo: formas de expressão criativas e comunicativas"

DOSSIER: "World's lectures: forms of expression creativity and communication"

Rodas culturais, UPP, funk e milícias: uma análise da cultura urbana carioca frente às políticas de segurança e às organizações criminosas

Cultural Circles, UPP, Funk and Militia: an analysis of Rio de Janeiro's urban culture in relation to security policies and criminal organizations

ROSSI GONÇALVES e GUILHERME SANTOS

O sarau como estratégia de resistência poética e reflexão sobre novos territórios culturais

Soiree as poetic resistance strategy and reflection on cultural new territories

IDEMBURGO FRAZÃO

Torcedores organizados: enigma como contrapeso

ao fantasma da razão esclarecida

Football supporters groups: enigma as counterweight

to the ghost of the enlightened reason

GUSTAVO COELHO

Cidade Maravilhosa: O Rio de Janeiro representado pelas letras

Wonderful City: Rio de Janeiro represented by the letters

PRISCILLA OLIVEIRA XAVIER

Práticas de leitura na contemporaneidade:

experiências em bibliotecas na cidade do Rio de Janeiro

Reading practices in contemporaneity:

libraries' experiences in the city of Rio de Janeiro 
Mudar de corpo em ode aos invisíveis

Changing body in ode to the invisible ones

ANA HUPE

Carnet de routes: following Blaise Cendrars in Brazil

Diário de bordo: seguindo Blaise Cendrars no Brasil

LUCA FORCUCCI

Espetáculo "O2": aproximações entre a prática de aula e a criação artística

"O2" show: approximation between class practice and artistic creation

LARA SEIDLER DE OLIVEIRA

ARTIGOS / ARTICLES

Cultura: a dádiva da sociedade

Culture: the social's gift

JÚLIO AURÉLIO VIANNA LOPES

O jornalista e o assessor de imprensa no cinema noir através do filme $A$ Embriaguez do Sucesso

The Journalist and the PR professional in Film Noir in the movie Sweet Smell of Success ALEX SAMPAIO PIRES E MICHELE NEGRINI

Seguindo a constituição da Joalheria contemporânea

Following the constitution of the Contemporary Jewelery

ANA NEUZA BOTELHO VIDELA

Experimentos Audiovisuais entre Ramagens Otonianas: uma leitura ecossistêmica comunicacional sobre a representação da Amazônia na arte de Otoni Mesquita

Creative ramifications: An ecosystem reading on the Amazon by Art Otoni Mesquita ÍTALA CLAY DE OLIVEIRA FREITAS E RAFAEL DE FIGUEIREDO LOPES

Veredas abertas da América Latina 